\title{
An Improved Method for Error Analysis of Intelligent Distribution Terminal
}

\author{
Wen-bo Fan ${ }^{1}$, Si-ran Zuo ${ }^{2^{*}}$, Jin-wei Fu ${ }^{1}$ and Zhong-yu Wang ${ }^{2}$ \\ ${ }^{1}$ Distribution Research Department, China Electric Power Research Institute, Beijing 100192, China \\ ${ }^{2}$ School of Instrumentation Science and Opto-Electronics Engineering, Beihang University, Beijing \\ 100191, China
}

Keywords: Error Analysis, Intelligent Distribution Terminal, Maximum Entropy, Measurement Uncertainty.

\begin{abstract}
An improved maximum entropy method is proposed to detect the error for intelligent distribution terminal. Firstly, a constraint maximum entropy function of the measurement error is established by introducing the uncertainty of the detected data in the intelligent distribution terminal. Besides, the Lagrange multipliers of this function are improved by loop iterations. Subsequently, the error of intelligent distribution terminal is detected by parameter update strategy of the maximum entropy method. Finally, an electrostatic discharge test is carried out with the proposed method under the electromagnetic compatibility conditions. The experimental results show that this method is more suitable for error analysis in intelligent distribution terminal than conventional maximum entropy method and the Botev kernel density estimation method.
\end{abstract}

\section{Introduction}

Power distribution automation is an important mean to achieve fast fault location, fault isolation and service restoration. The distribution automation (DA) system is comprised of the main station, the sub-stations and the intelligent distribution terminal [1]. This system is used to monitor, coordinate and control the distribution equipment in real time. With the rapid development of the distribution network construction, automatic detection technology of the DA system has drawn much attention in recent years.

In the error analysis field, the measurement uncertainty is often used to assess the quality of the observed data. The power data of the primary equipment in the distribution network are collected by the intelligent distribution terminal, and the results are displayed by the main station in a DA system. Therefore, the measurement uncertainty and error distribution of the intelligent distribution terminal are the foundations for assessing the quality of the observed data. The commonly used methods for solving the error distribution of the terminal are the kernel density estimation methods such as the Botev kernel density estimation method [2]. The Botev kernel density estimation method has a good estimation ability, and does not require for any additional assumptions of data distribution. Nevertheless, the choice of bandwidth depends heavily on the higher order terms of kernel function when the kernel density estimation of diffusion equation is built. For a complexity DA system, the Botev method has poor applicability in practice due to its slow convergent speed.

The maximum entropy method can achieve the unbiased estimation of the observed data [3]. It has been widely applied into the estimation of data distribution of various detection systems recently. Meng [4] estimated prior probability of faults according to maximum entropy principle. $\mathrm{Lu}$ [5] optimized the 2 dimensions PID controller parameters by adopting the maximum entropy distribution algorithm. Yari [6] used the long-term data for the average daily wind speed to examine the Rayleigh distribution to confirm the accuracy and efficiency of the proposed maximum entropy algorithm. These previous methods directly took the multi-order origin moment and the second order central moment of discrete data as the constraint conditions. However, the influences of the concrete detection method and detection equipment have been not considered. These inevitably lead to the low estimation accuracy.

In this paper, an improved maximum entropy detection method is proposed to estimate the measurement error probability density function of the intelligent distribution terminal. Maximum 
entropy distribution function is established and the corresponding parameters are estimated by the update strategy. In addition, the comparisons of the proposed method with the conventional maximum entropy method and the Botev kernel density estimation method are conducted with an electrostatic discharge test. The results demonstrate the feasibility and validity of the improved method.

\section{Improved Maximum Entropy Method}

The probability density function of the observed data of intelligent distribution terminal is usually continuous. Therefore, the corresponding measurement error $x$ should also be a continuous random variable. According to the maximum entropy principle, the relationship between the information entropy $h(x)$ and probability density function $p(x)$ of $x$ satisfies following formulas [7]

$$
\max (h(x))=-\int p(x) \log p(x) \mathrm{d} x .
$$

Actually, both multi-order original moment and second order central moment of $\mathrm{X}$ could be considered as the constraint conditions. But these constraint conditions have a higher requirement for the detected data, which should have a large sample size and be acquired under a low noise environment. In fact, the actual detection environment is always affected by the large noise. Therefore, it is necessary to explore the new constraint conditions for improving the performance of the conventional method.

Uncertainty $U$ of the measurement error includes direct and indirect information of the observed data, which reflects the detected data more deeply. So the $U$ of the measurement error could be considered as a constraint condition. According to the definition of uncertainty, the relationship between probability density function $\mathrm{p}(\mathrm{x})$ and $\mathrm{U}$ is built [8].

$$
\int_{\bar{x}-U}^{\bar{x}+U} p(x) \mathrm{d} x=p, \text { with } \bar{x}=\int_{-\infty}^{\infty} p(x) x \mathrm{~d} x
$$

And a constructed piecewise function $g(x)$ is used to adjust the upper and lower bounds of the integral as

$$
g(x)=\left\{\begin{array}{ll}
0 & x<\bar{x}-U, \quad x>\bar{x}+U \\
1 & \bar{x}-U \leq x \leq \bar{x}+U
\end{array} .\right.
$$

After adjustment, Eq. 2 can be modified to

$$
\int_{-\infty}^{\infty} p(x) g(x) \mathrm{d} x=p
$$

Thus, the improved maximum entropy can be presented as

$$
\max (H(x))=-\int_{-\infty}^{\infty} p(x) \log p(x) \mathrm{d} x, \text { with } \int_{-\infty}^{\infty} p(x) g(x) \mathrm{d} x=p
$$

For the convenience of further calculations, Lagrange multipliers $\lambda_{0}, \lambda_{1}, \cdots, \lambda_{n}$ are adopted. A function $\mathrm{F}$ is constructed as

$$
F=h(x)+\left(\lambda_{0}+1\right)\left(\int p(x) \mathrm{d} x-1\right)+\sum_{i=1}^{n} \lambda_{i}\left(\int p(x) f_{i}(x) \mathrm{d} x-\mu_{i}\right)
$$


Let $\partial F / \partial p(x)=0$, the probability density function $\mathrm{p}(\mathrm{x})$ can be expressed as :

$$
p(x)=\exp \left(\lambda_{0}+\sum_{i=1}^{n} \lambda_{i} f_{i}(x)\right)
$$

Based on Eq. 5, the maximum entropy probability density function of $\mathrm{x}$ can be written as :

$$
p(x)=\exp \left(\lambda_{0}+\lambda_{1} x+\lambda_{2}(x-\bar{x})^{2}+\lambda_{3} g(x)\right) .
$$

The residual error sum $r$ is defined as :

$$
r=\sum_{i=1}^{n} r_{i}^{2} \rightarrow 0
$$

Where ${ }^{r_{i}}$ is the improved residual error which represents the convergent degree of ${ }^{\mu_{i}}$. The lower the value of $r_{i}$ is, the higher convergent degree of $\mu_{i}$ is and the better improved efficiency of $\lambda_{0}, \lambda_{1}, \cdots, \lambda_{n}$ is. When Eq. 9 satisfies the convergent conditions, the corresponding Lagrange multipliers $\lambda_{0}, \lambda_{1}, \cdots, \lambda_{n}$ should be the results of optimization and then $\mathrm{p}(\mathrm{x})$ in Eq. 8 can be calculated.

\section{Experiment and Data Analysis}

An electromagnetic compatibility test is carried out to validate the performance of the proposed method. The error probability density function of the observed data from intelligent distribution terminal can be solved quantitatively. The measurement results of the test are shown in Fig. 1. In theory, the ideal output of the intelligent distribution terminal is an approximate step signal, while the actual collected signal is affected by environmental noise. From Fig. 1, the detected data from 0 to $20 \mathrm{~ms}$ fluctuates much more violently than the data from $20 \mathrm{~ms}$ to 100 . It is mainly because of the touch point discharging with instantaneous large current, which forms the interference to intelligent distribution terminal.

The measurement uncertainty of the intelligent distribution terminal in the test is evaluated with 95\% confidence probability. There are two main aspects of the sources of the measurement uncertainty: one is the type A standard uncertainty affected by temperature and humidity in the environment and represented by repeatability of measurement. The other is the type B standard uncertainty which reflects the measurement accuracy of oscilloscope used in the test and the accuracy of electrostatic discharge gun's output voltage.

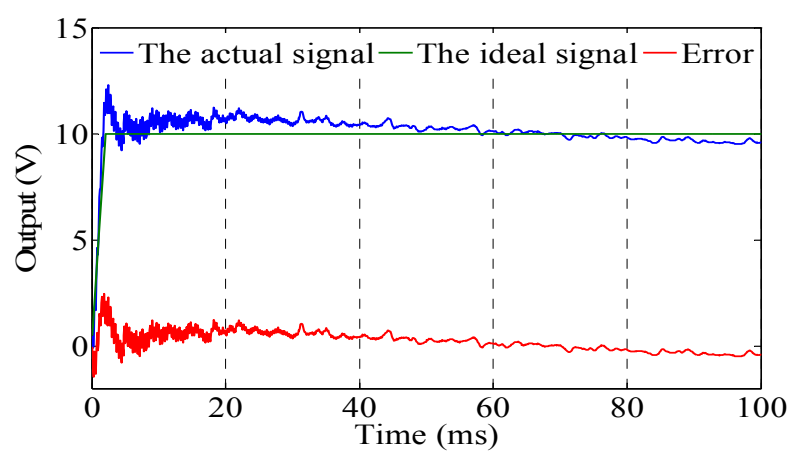

Figure 1. Measurement results

A total of 1000 data are adopted, and the value of type A standard uncertainty is calculated as $\mathrm{u} 1=0.151 \mathrm{~V}$. According to verification certificate of oscilloscope, the value of type B standard 
uncertainty is calculated as $\mathrm{u} 2=0.032 \mathrm{~V}$. Consequently, the combined standard uncertainty and the expanded uncertainty of the measurement error are obtained. The values of these two uncertainties are $0.154 \mathrm{~V}$ and $0.308 \mathrm{~V}$, respectively.

The expanded uncertainty evaluation result of the measurement error is substituted into Eq. 2 . The minimum residual error sum is set as $\mathrm{rmin}=10-4$, then the improved results of Lagrange multipliers are listed in Table 1.

Table 1. Improved results of Lagrange multiplier

\begin{tabular}{|c|c|c|c|c|}
\hline Lagrange multiplier & $\lambda_{0}$ & $\lambda_{1}$ & $\lambda_{2}$ & $\lambda_{3}$ \\
\hline Improved results & -1.383 & 0.1248 & -6.785 & 0.09 \\
\hline
\end{tabular}

Additionally, the conventional maximum entropy method and the Botev method are respectively adopted to solve the probability density function with the same measurement data [9]. The comparison of different distribution methods is displayed in Fig. 2. According to Fig. 2, the proposed method is consistent with the Botev method completely, and the former can effectively eliminate the volatility effects occurring in boundary where the probability density is smaller. Moreover, the probability distribution curve generated by the proposed method has a higher maximum peak and is steeper than that generated by the conventional maximum entropy method. This kind of characteristic illustrates that the proposed method can greatly avoid over-smoothness and has a better anti-noise ability.

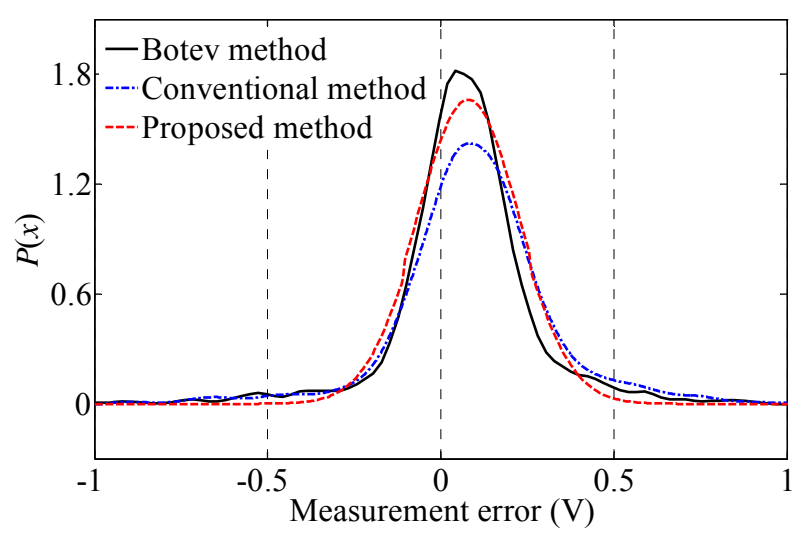

Figure 2. Comparison of different distribution methods

To compare the evaluation accuracy of the above three methods, the standard deviations are calculated. In addition, by taking the Botev method as the standard [9], the relative standard deviations of the proposed method and the conventional method are shown in Table 2 . The relative standard deviation of the proposed method is less than $5 \%$, which illustrates the correctness and validity of this method. Besides, the relative standard deviation of the proposed method is decreased by $4.92 \%$ smaller than that of the conventional method.

Table 2. Calculative results and error analysis

\begin{tabular}{|l|l|l|}
\hline Methods & Standard Deviation (V) & Relative Standard Deviation \\
\hline Botev Method & 0.1604 & - \\
\hline Conventional Method & 0.1458 & $9.10 \%$ \\
\hline Proposed Method & 0.1537 & $4.18 \%$ \\
\hline
\end{tabular}


Under the same conditions, the number of iterations and the run time of the proposed method and the Botev method are listed in Table 3. The number of iterations of the proposed method is less than half that of the Botev method. It can be seen that the computing efficiency of the proposed method improved nearly two times than the Botev method.

Table 3. Comparison of the Botev Method and the proposed method

\begin{tabular}{|c|c|c|}
\hline Methods & The number of iterations & The run time (s) \\
\hline Botev Method & 258 & 31.98 \\
\hline Proposed Method & 122 & 16.05 \\
\hline
\end{tabular}

\section{Conclusions}

1) This paper proposes an improved maximum entropy method for solving the measurement error probability distribution by introducing the uncertainty as one of constraint conditions into maximum entropy.

2) The evaluation accuracy of the proposed method is improved by $54 \%$ higher than that of the conventional maximum entropy method. This result demonstrates the correctness and validity of the proposed method.

3) The proposed method has less number of iterations, and the computing efficiency improved nearly two times than the Botev method. It illustrates the proposed method is more suitable for intelligent distribution terminal installed in complex environments.

\section{Acknowledgement}

This research was financially supported by "Research on establishment method of accuracy loss model for distribution automation test system".

\section{References}

[1] CONG Wei, LU Qing-dong, TIAN Chong-wen, et al. Smart distribution terminal unit and its standardized modeling, Automation of Electric Power Systems, 37(10) (2013) 6-12.

[2] A. Balestrino, A. Caiti, A. Noe'. Maximum entropy based numerical algorithms for approximation of probability density functions, European Control Conference (ECC), (2003)

[3] V.F. Ruiz. An efficient approach to highly non-linear estimation. International Conference on Digital Signal Processing, (2002).

[4] MENG Xiao-feng, JI Hong, WANG Guo-hua, et al. Maximum entropy method for fault prior probabilities. Journal of Beijing University of Aeronautics and Astronautics, 32(11) (2006) 1320-1323.

[5] Lin L. Optimal design for 2-DOF PID controller based on Maximum Entropy Estimation of Distribution Algorithm, International Conference on Intelligent System and Knowledge Engineering. IEEE, (2008) 1338-1342.

[6] Yari G, Farsani Z A. Application of the Maximum Entropy Method for Determining a Sensitive Distribution in the Renewable Energy Systems, Journal of Energy Resources Technology, 137(4) (2015).

[7] Ke Zeng, Hua-qiang Li, Meng-Jie Zeng.Power system risk security assessment based on maximum information entropy principle. IEEE International Conference on Power System Technology, (2014) $421-426$. 
[8] ZHU Jian-min, GUO Bing-jing, WANG Zhong-yu, et al. Study on evaluation of measurement result and uncertainty based on maximum entropy method, Electrical Measurement \& Instrumentation, 42(8) (2005) 5-8.

[9] Giantomassi A, Ferracuti F, Iarlori S, et al. Electric Motor Fault Detection and Diagnosis by Kernel Density Estimation and Kullback-Leibler Divergence Based on Stator Current Measurements, IEEE Transactions on Industrial Electronics, 62(3) (2015) 1770-1780. 